\title{
Developing and Validating a Fireworks-Related Syndrome Definition in Kansas
}

\author{
Zachary M. Stein* \\ Bureau of Epidemiology and Public Health Informatics, Kansas Department of Health and Environment, Topeka, KS, USA
}

\section{Objective}

To develop a syndrome definition and analyze syndromic surveillance data usefulness in surveillance of firework-related emergency department visits in Kansas.

\section{Introduction}

Across the U.S.A., multiple people seek treatment for fireworksrelated injuries around the July $4^{\text {th }}$ holiday. Syndromic surveillance in Kansas allows for near real-time analysis of the injuries occurring during the firework selling season. During the 2017 July $4^{\text {th }}$ holiday, the Kansas Syndromic Surveillance Program (KSSP) production data feed received data from 88 EDs at excellent quality and timeliness. Previous and current firework safety messaging in Kansas is dependent on voluntary reporting from hospitals across the state. With widespread coverage of EDs by KSSP, data can be more complete and timely to better drive analysis and public information.

\section{Methods}

KSSP data was queried through the Electronic Surveillance System for the Early Notification of Community-based Epidemics (ESSENCE) v.1.20 provided by the National Syndromic Surveillance Program. Data between June 12, 2017 and August 13, 2017 were queried. The first query (Query A, Table 1.) searched the Discharge Diagnosis History field for the "W39" ICD-10 Diagnosis code, "Discharge of firework." These records were searched for common firework terms contained in the Chief Complaint History field. These firework-related free text terms (Query B, Table 1.) were then combined with other potential firework-related terms to create a preliminary free text query (Query C, Table 1.). This preliminary query was run on the Chief Complaint History field. Data were then searched for false positive cases and appropriate negation terms were included to accommodate this. The new query with negation terms (Query D, Table 1.) was run on the Chief Complaint History field, combined with the results from the Discharge Diagnosis History field, and then combined records were de-duplicated based on a unique visit identifier. The final data set was then classified by the anatomical location of the injury and the gender and age group of the patient.

\section{Results}

The initial query (Query A, Table 1.) for the diagnosis code "W39" returned 101 unique ED visits. Of these 101 unique ED visits, the following terms were identified in the Chief Complaint History field: shell, artillery, bomb, sparkler, grenade, fire cracker, firework, and firework show. These key terms were translated into Query B, Table 1. Other key terms deemed likely to capture specific firework-related exposures were then included into Query C, Table 1., including roman, candle, lighter, M80, and punk. Query C was then used to query the Chief Complaint History field, returning 144 unique ED visits. Cases captured by Query $\mathrm{C}$ were then reviewed by hand for false positives and the negation terms, lighter fluid, fish, nut, and pistachio, were incorporated the Query D, Table 1. The previous process for Query $\mathrm{C}$ was then repeated on Query D, leaving a remaining 136 unique cases. Query A's 101 unique ED visits was then combined with the 136 unique ED visits captured by Query D and de-duplicated. The de-duplicated data set contained 170 unique
ED visits which were then reviewed by hand for false positives. The final removal of false positives from the combined and de-duplicated data set left a remaining 154 unique ED visits for firework-related injuries during this time period.

For these data, the most common victims of firework injuries were males, accounting for $65.5 \%$ of all firework related ED visits and children ages 0 to 19 accounting for $44.2 \%$ of these visits. At every age breakout, male injuries exceeded female injuries. The most common anatomical location of the injury was one or both hands with $38.3 \%$ of all injuries mentioned hands as their primary injury. Injuries to the eyes, face, and head accounted for the second most injuries ( $28.6 \%$ of all patients).

\section{Conclusions}

The selling of fireworks will be a yearly occurrence of a specific exposure that can potentially lead to injuries. Utilizing syndromic surveillance to review the holiday firework injuries is a very rapid method to assess the impact of these injuries and may allow for future direction of public information during the holiday. Having a syndrome definition that builds on knowledge from previous years will allow for quicker case identification as well.

State public information regarding firework safety can be significantly bolstered by accurate and rapid data assessment. Developing a firework injury syndrome definition that is accurate and returns information rapidly has allowed for increased buy-in to the Kansas Syndromic Surveillance Program from public information offices, fire marshal's offices, and other program fields.

Queries: Firework-Related Injuries

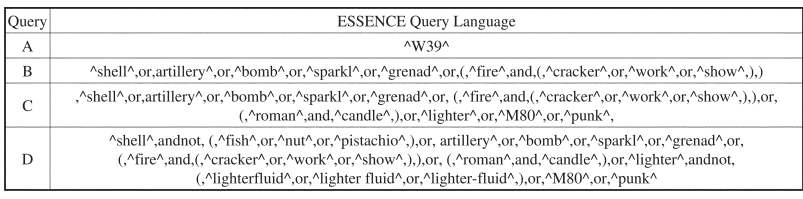

\section{Keywords}

Firework; Kansas; Injury; Syndromic; Surveillance

\section{Acknowledgments}

Data collection was supported by the Grant or Cooperative Agreement Number 1 U50 OE000069-01, funded by the Centers for Disease Control and Prevention. Its contents are solely the responsibility of the authors and do not necessarily represent the official views of the Centers for Disease Control and Prevention or the Department of Health and Human Services

\section{*Zachary M. Stein}

E-mail: zstein@kdheks.gov 